\title{
Development of Heating Power Station Process Mathematical Model for Personnel Training Simulator
}

\author{
Sholpan M. Baimatayeva \\ Bulat Khissarov \\ Aksholpan A. Kopesbayeva
}

Email address: b_shm@mail.ru

\section{Doi:10.5901/mjss.2014.v5n23p1490}

\begin{abstract}
This paper is devoted to study of models for operator training systems based on a simulator. Situational modeling method, allowing to develop technological process control skills at qualitative level, was applied for demonstration of process situations. A procedure for object control was developed for the operator's training system, which is based on models of operator's activity, allowing to simulate a problem situation and to develop a control decision with an application of situation control expert system concepts.
\end{abstract}

Keywords: simulator, steam generator, situation, operating process

\section{Introduction}

Emergence of complex production technologies and toughening of requirements to reliability and safety of heating power station operation demands improvement of production process control quality. One of the important factors of control quality improvement is qualification of operation personnel, the sufficient level maintenance of which is provided by specialized systems of operators training based on simulators.

The main task preceding to development of operators training systems based on a simulator is working out of mathematical model of an object. Considering influence of parallel operating units the models of a high-pressure general steam conduit and a feed water line for cross-connection heating power plants are of essential value [1]. A steam generator is connected to the units the processes in which are characterized by a heat exchange between the heating medium and a running coolant. The peculiarity of these processes lies in density influence on the flow internal structure, i.e. on temperature, pressure and speed.

Methods of solution of operators' decision-making skill development problem are represented by mathematical models of control process simulation. The most developed models demonstrating processes of a water-steam circuit are represented by systems of differential equations with partial differential coefficients and systems of thermal and mass balance equations [2-6]. Some processes are described by a system of non-linear algebraic equations [7]. Logic and logic- dynamic models which demonstrate different modes are applied for simulation purposes. As a rule, logic- dynamic models are non-linear ones and require application of differential equations with higher-order partial differential coefficients.

Nonlinear models describing water-steam circuit processes with distributed constants provide with more exact quantitative and qualitative information about the steam generator process control modes if no time limits are set for computational process [8]. There can be introduced a definition of maximum circuit calculation time - it is a time at which the trainee starts to distinguish visually the time delays arising at creation by him perturbing actions in simulator operations. At exceeding such time limit the simulator becomes ill-controlled.

One of the turning points in development of numerical nonlinear model is a selection of equations solution method. Application of numerical integration is connected with loss of solution stability. As it is marked in work [9]: "... For complex systems with a great number of integrated variables and feedbacks, including cross feedbacks, the most radical way of stability provision remains to be an integration step reduction in the timeframes what leads to increase in machine time expenditures and toughening of requirements to computer performance", what is not a serious problem for currently produced state-of-the-art computer hardware. 
Besides, in real operation conditions the operator has tasks set with malfunctions and failures of equipment. Therefore, it is necessary to define the most expedient control actions to be taken by the operating personnel in such situations. Solution of the specified problem can be found by application of situational modeling methods allowing to develop technological process control skills at qualitative level.

\section{Operator's ACtion Model as a Control Function Performance}

Thermodynamic cycle of power station is performed under the following flowchart. Fuel is fed through burners to a furnace where it is burnt by a flare method. For maintenance of burning process the air is supplied to the furnace. The combustion gases formed in the course of burning are sucked off by an exhaust blower. On their way they pass through heating surface areas of superheaters, feed-water economizer, air heater. Steam production process runs in ascending pipes of a water-flow circuit. From the drum the saturated steam is supplied to a steam superheater, where it is heated up to the set temperature due to flare radiation and convective heating by fuel gases (Fig. 1.):

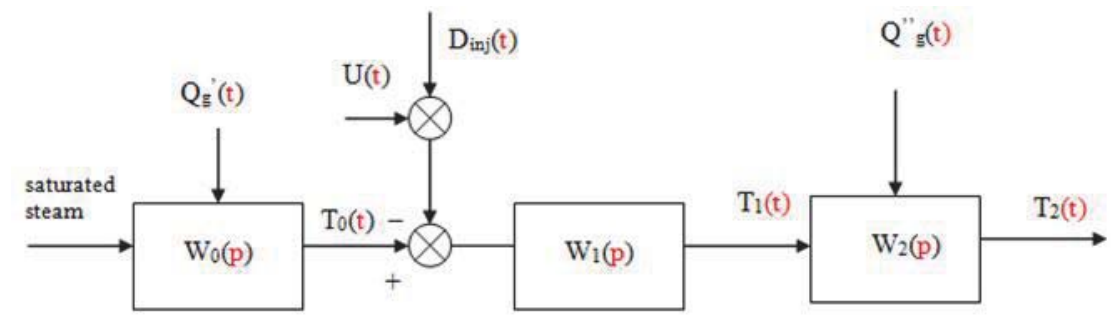

Fig.1. Steam generator structural flowchart

Symbols in the figure: $Q_{g}^{\prime}(t)$ - fuel gas flow rate; $D_{\text {inj }}(t)$ - injection water flow rate; $T_{0}(t)$ - coolant temperature in front of injection; $T_{1}(t)$ - coolant temperature behind injection; $T_{2}(t)$ - superheated steam temperature; $W_{0}(p), W_{1}(p), W_{2}(p)$ transfer functions.

Transfer functions represent a combination of the first order aperiodic element and a lag element. Steam superheating temperature is adjusted in a desuperheater by injection. Main controlled values of the boiler are consumption, pressure and temperature of a superheated steam. Steam consumption is a variable, and its pressure and temperature are maintained within maximum deviations. The listed values change as a result of control actions and under influence of external and internal perturbing actions.

Based on the analysis of operator's activity regarding the complex process systems control there was developed a functional model of the controlled object. Functionally, the simulation object consists of the following elements: a controlled object and operational procedures, control elements; an information field showing the equipment status and operational procedure parameters values. The most efficient method of the operating personnel professional training is training based on a simulator with physical or functional model of the controlled object. Moreover, it is necessary to develop a training scenario consisting of the following steps: narrative injection for a trainee; commenting of actions; inclusion of an object of malfunctions and perturbing actions into a simulator; monitoring of the controlled object simulator condition; estimation of certain actions [10]. Each of separate training exercises can be considered as a separate state of training $S T_{i}$. In its turn, each training exercise may be presented in the form of a unique sequence $S U_{i}$, each of which is described by standard actions of the trainer. Points of the oriented graph scenario of training will be the states of training exercises $S U_{i}$, edges of graphs will be represented by decision-making on transition to another state of the training exercise ${ }^{P R_{i}}$. A scenario of training may be shown as an oriented graph (Fig. 2): 


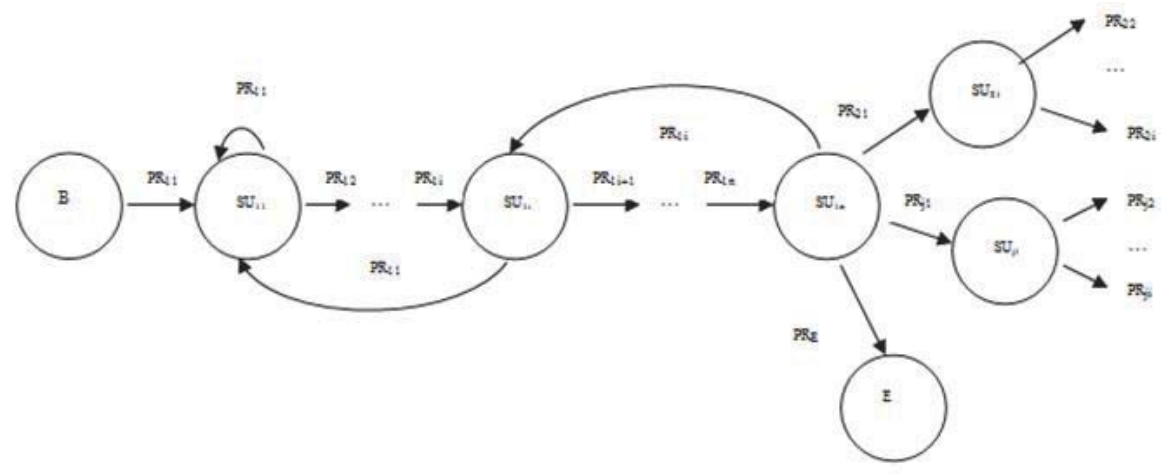

Fig. 2. Scenario of training

For simulator operation there was selected a zone of boiler feeding with water, as one of the most important zones in operational control of cross-connection heat power plants and as a zone with a whole set of typical production units used at development of a simulator - fittings, mechanisms, parameters, automatic controllers, alarm system, security and interlock systems. Initial materials were represented by heat power plants flow diagrams, design materials for personnel training simulators and station documents on equipment operation. Besides, there were gathered materials on operational characteristic of equipment and elements of the simulated flow diagrams, static and dynamic characteristics.

Based on the operator's activity analysis, the simulated situations were defined in a water-steam circuit, physical assumptions were made and a model development order was established. Condition of an actuating medium along a water-steam circuit is described by equations of energy, mass conservation (continuity), motion and state. The energy equation establishes that the difference between a heat inflow and a heat outflow goes into change of heat energy enclosed in steam, water and metal spaces [11]:

$$
D_{\mathrm{B} 1} i_{1}-D_{B 1}{ }^{i}+Q=V \frac{d \rho}{d \tau},(1)
$$

where $D_{B 1}$ is medium consumption at the input $(\mathrm{kg} / \mathrm{s} ; \mathrm{t} / \mathrm{h}) ;{ }_{1}, i$ mean entalpy at the input and output, respectively $(\mathrm{kkal} / \mathrm{kg}) ; \mathrm{Q}$ is a thermal flow $(\mathrm{kW}) ; \tau$ means time $(\mathrm{s}) ; \mathrm{V}$ is medium volume in the area $\left(\mathrm{m}^{3}\right), \rho$ is density $\left(\mathrm{kg} / \mathrm{m}^{3}\right)$.

Continuity equation shows that the difference between the inflow and outflow is equaled to the substance volume change in steam and water spaces:

$$
D_{B 1}-D=V \frac{d \rho}{d \tau},(2)
$$

Motion equation resolves into relationship between differential pressure in an inlet chamber and in a steam-supply pipe, and consumption:

$$
p_{1}-p=\xi \frac{D^{2}}{\rho}
$$
coefficient.

where $\mathrm{p}_{1}, \mathrm{p}$ mean medium pressure in front of and behind the resistance $\left(\mathrm{kgf} / \mathrm{cm}^{2} ; \mathrm{MPa}\right) ; \xi$ is hydraulic friction

Equation of state:

$$
\begin{aligned}
& \rho=\rho(p, t)(4) \\
& i=i(p, t) \quad(5)
\end{aligned}
$$

For solution of control problems in training systems at formalisation of a real system, a number of assumptions and simplifications are made at description of a dynamic model. An assumption about one-dimensionality of an actuating medium flow was taken as a basic assumption. Characteristics of areas are set by the parameters defining condition of the medium (pressure, consumption, temperature) at the input and output area. Other parameters depend on design features of such area and thermophysical data (heat capacity of metal, pipeline inner surface, heat transfer coefficient of pipeline inner surface and etc.).

Thermal channels of a superheater with convectional-radiation heat supply may be described by the equation of energy and heat balance. Heat balance equation is used for determination of temperature of a heating surface area pipe wall. Heat flow from the wall to an actuating medium is defined by the following relationship:

$$
Q=\alpha_{B} H_{B}(\theta-t),(6)
$$


where ${ }_{B}$ is a heating internal surface $\left(\mathrm{m}^{2}\right) ; \alpha_{B}$ means a heat-transfer coefficient of pipeline inner surface $\left[\mathrm{kkal} /\left(\mathrm{m}^{2} \cdot \mathrm{h} \cdot{ }^{\circ} \mathrm{C}\right)\right] ;$ is a separating wall temperature $\left({ }^{\circ} \mathrm{C}\right) ; t$ is an actuating medium temperature $\left({ }^{\circ} \mathrm{C}\right)$. Steam generator model is based on a system of linearized original nonlinear equations.

Study of the model validity is made on the ground of thermal test data provided by the heat automation workshop of Almaty heating power plant-2 according to the method described in [12].

Difference in dynamic characteristics may be brought to change of amplification factor $\mathrm{K}$, time-scale factor a and a transient process form. First two characteristics are described by the following transformations:

$$
\begin{aligned}
& y^{\prime}(\tau)=K y(\tau)(7) \\
& y^{\prime}(\tau)=y(a \tau)
\end{aligned}
$$

where y, y' mean initial and transformed processes, respectively. formula:

Statical accuracy of a tentative model in singular points (start, end, extreme points) is defined by the following

$$
\left|y_{j}-y_{j}^{M}\right| \leq \sqrt{\sigma_{c m j}^{2}+0,25 \Delta^{2}}
$$

where $\sigma_{\text {cmj }}^{2}$ is a dispersion of a reference value in a singular point $j ;{ }^{y, y^{M}}$ mean processes in a reference and a tentative model at the same perturbing action; $\Delta$ - unit of the last order. There were considered the processes the singular point static error of which did not exceed the specified value.

At time scale variance a measure of process deviation $v$ may be set by the following relation

$v=\Delta a=a^{M}-1,(10)$

where $a^{M}$ means change in a time scale which is better brings the model into proximity based on minimum condition of relationship:

$\int^{T}\left[y(a \tau)-y^{M}(\tau)\right]^{2} d \tau$

0

where ${ }^{T}$ is time of process observation by a probationer.

At determination of dynamic accuracy with due consideration of random factors the following normal probability law is assumed:

$f(v)=\frac{1}{\sqrt{2 \pi} \delta} \exp \left(-v^{2} / 2 \delta^{2}\right)$ (12)

Adequacy level value is determined with an application of the following relationship:

$P(v)=F\left(\frac{v+\varepsilon}{\sigma}\right)-F\left(\frac{\nu-\varepsilon}{\sigma}\right),(13)$

where $\mathrm{F}$ is a normal distribution function.

\section{Steam Generator Mathematical Model Development for Training System}

Basic elements of a simulated object are represented by heating surfaces, non- heated areas, mixing areas (injections). Each element is described by the equations defining the relationship between input and output co-ordinates. Effects related to different character of paralleled line and shell processes are not taken into consideration. Mass and energy of all steam generator elements are assumed to be concentrated in a mass point. Thus, the parameters are assumed to be constant along the length in a tail end area, and area and input parameters agree with each other. Therefore, hydraulic friction is referred to the area outlet section and is considered as a local resistance. Input coordinates (environmental perturbing actions) are deviations from an initial steady state: feed water consumption; feed water temperature; crude fuel consumption; water consumption for each injection; positioning of secondary steam temperature controls. Output coordinates for the whole system are represented by deviations in consumption, pressure, temperatures, actuating medium and temperature of gases in boundary sections of elements. Transfer functions are defined by a sequential calculation of output deviations parameters of each area. Unit perturbing actions are delivered to the first area input, and output deviations of the previous area are delivered to an input of the next area.

Values of parameters necessary for calculation of equations coefficients are determined based on the initial data. Water and water steam thermodynamic property tables are used for determination of thermodynamic functions and their derivatives. 
Development of models of surface heat exchangers, steam space and injection collector as elements of a steam generator are given in [13]. For example, on the basis of shell and thermal balance energy equations the following system of the equations is obtained for deviations:

$$
\left\{\begin{array}{l}
\left(L_{B}+1\right) \Delta t-L_{B} \Delta t_{1}-T_{B} K_{p} \frac{d \Delta t}{d \tau}+T_{B} \frac{d \Delta t}{d \tau}-(1-n) K_{B} \Delta D_{B 1}=\Delta \theta \\
\Delta t+K_{Q} \Delta Q+n K_{B} \Delta D_{B 1}=T_{M} \frac{d \Delta \theta}{d \tau}+\Delta \theta
\end{array}\right.
$$

where $n$ is a parameter depending on washing type. The formula (14) has the following symbols for operating and design parameters:

$$
\begin{aligned}
& L_{B}=\frac{D_{B 0} c_{B}}{\alpha_{B 0} H_{B}}-\text { extension parameter; } \\
& T_{B}=\frac{G_{B} c_{B}}{\alpha_{B 0} H_{B}}-\text { heat accumulation in a coolant; } \\
& T_{M}=\frac{G_{M} c_{M}}{\alpha_{B 0} H_{B}}-\text { heat accumulation in metal; } \\
& K_{Q}=\frac{1}{\alpha_{B 0} H_{B}}, K_{B}=-L_{B} \frac{t_{0}-t_{10}}{D_{B 0}}, K_{p}=-\frac{\partial i / \partial p}{\partial i / \partial t} .
\end{aligned}
$$

Used symbols: ${ }_{B}{ }_{B}, c_{M}$ mean coolant and metal heat capacity, respectively $\left[\mathrm{kkal} /\left(\mathrm{kg}{ }^{\circ} \mathrm{C}\right)\right] ;{ }_{B}, G_{M}$ mean respective coolant and metal mass $[\mathrm{kg}] ;{ }^{t}, t\left[{ }^{\circ} \mathrm{C}\right]$ are temperatures at the input and output, respectively. Index "zero" characterizes parameters values in the initial steady-state mode, symbol $\Delta$ means parameters deviation. The abovementioned parameters represent complexes of operating and design parameters and determine a dynamic system inertia.

Equation for temperature deviation at the heat exchanger output obtained from (14) will be as follows:

$$
\Delta t=K_{t t 1} \Delta t_{1}+K_{t Q} \Delta Q+K_{t D B 1} \Delta D_{B 1}+K_{t p 1} \Delta p_{1} \text {, (15) }
$$

In the equation (15) coefficients ${ }^{K} t 1,{ }_{t Q},{ }_{t D}{ }_{B 1}, K_{t p 1}$ are equal to partial derivative values in reference points of steady-state characteristic relationship: "output temperature - input temperature", "output temperature - heat load", "output temperature - steam consumption", "output temperature - input pressure", respectively.

Dependence of an output variable at a stepwise change of components in the right side of an equation is an exponent with a time constant and is notable for transfer factors for input parameters values. In order to simulate characteristics of channels for exposure transfer from input values to output values, there must be calculated coefficients based on heat calculations data [14]. Depending on the operational task character the number of environmental perturbations is defined and is referred to a category of variables of equation system describing the controlled object. A software was developed for steam generator operation simulating based on application of certain elements coefficient matrix. At software program use the area characteristics are set by seven parameters: feed water temperature $\left({ }^{t_{-}} f w\right.$, fuel consumption $(W)$, feed water consumption ( $\mathrm{g}_{-} \mathrm{fw}$ ), injection water consumption ( $\mathrm{g}_{-}{ }^{\mathrm{inj}}$ ), superheated steam temperature $\left({ }^{\mathrm{t}}{ }^{\mathrm{ss}}\right)$, superheated steam pressure $\left(\mathrm{p}^{\mathrm{p}} \mathrm{ss}\right)$, superheated steam consumption ( $\left.\mathrm{g}_{-} \mathrm{ss}\right)$.

Algorithmic part provides a possibility for introduction of perturbing actions by a trainer and control actions by a trainee according to which the new values of parameters must be determined [15]. The system consists of separate interlinked elements, each of which is described by an operator which determines its output $Y$ and current state at an input $X$ and previous state. Interrelation of elements is described by an operator as $Y=W X$, where $w$ is a matrix of coefficients. Functioning of automated units is thus considered. The relevant transition function ${ }^{h_{i j}(t)}$ can be obtained at sudden change of input parameters or by solution of differential equation system, or by an experimental method. By multiplying the relevant element of matrix $\mathrm{W}$ by $h_{i j}(\tau)$, for example, like: $h_{i j}(\tau)=e^{-\tau} / T_{j}$, or $h_{i j}(\tau)=1-e^{-\tau} / T_{j}$ there can be considered a dynamics of the $\mathrm{i}$ - parameter change influence by deviation of $\mathrm{j}$ - parameter, for which $\mathrm{T}_{\mathrm{j}}$ depends on system parameters. Software operation algorithm is given in Fig. 3: 


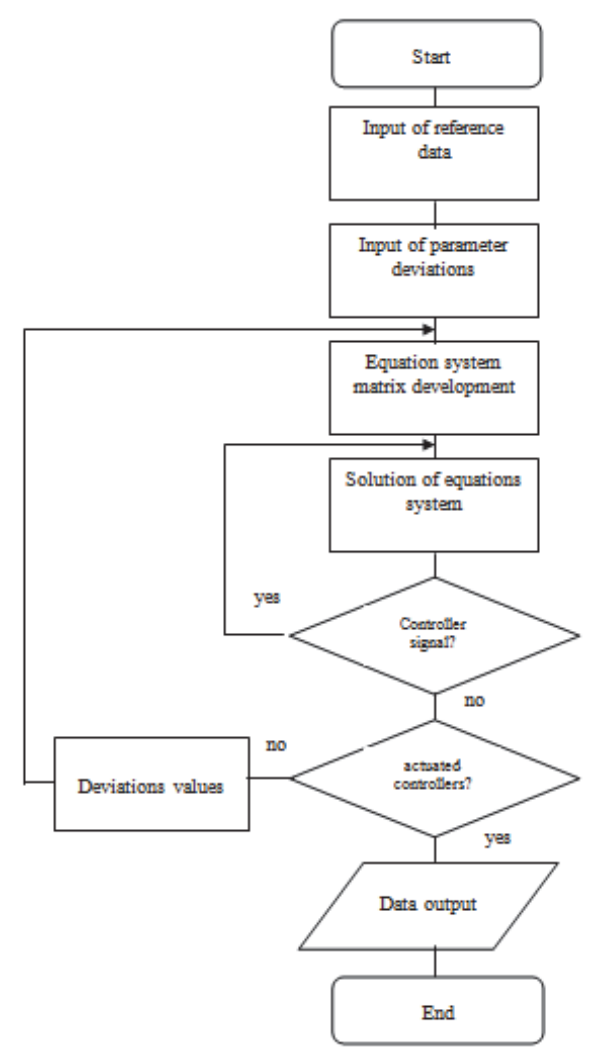

Fig. 3. Software operation algorithm

\section{Situation Analysis of Steam Generator Modes}

In the course of equipment operation there often occurs a situation when the operator cannot make an optimal decision by a reason of too much interlinked parameters. The operator has tasks including malfunctions and failures of equipment. In such cases the operator appears to be in a situation demanding making a choice for determination of reasonable control actions [16]. The process unit operation can be described by a certain combination of situations. For example, at overheated steam temperature control, being limited only to three parameters - at change of fuel consumption from 110 $\mathrm{kg} / \mathrm{s}$ to $130 \mathrm{~kg} / \mathrm{s}$ with an increment of $1 \mathrm{~kg} / \mathrm{s}$, feed water consumption from $400 \mathrm{~kg} / \mathrm{s}$ to $420 \mathrm{~kg} / \mathrm{s}$ with an increment of 1 $\mathrm{kg} / \mathrm{s}$, steam temperature from $530^{\circ} \mathrm{C}$ to $540^{\circ} \mathrm{C}$ with an increment of $1^{\circ} \mathrm{C}$, there appears to be a great number of situations. Meanwhile, from the point of view of the requirements to the controlled object, these situations are approximately the same and describe normal operation of the unit. An approach, which takes into account basic conditions of the controlled object, should be selected. Proceeding from this, there should be determined an interval of measured parameters acceptable value based on the results of the controlled object study (BKZ-420-130-7c type steam generator) at Almaty heat power plant-2 carried out in Kazenergonaladka company. As a result of cause-effect relationship study and determination of sequential analysis of probable process situations there were revealed the most often occurring situations which represent a data for situational model functioning. Meanwhile, development of a problem situation and working out of optimal control solutions which are determined by selection of the best control strategy make the basis for operator's training.

Training management is carried out in two stages: setting of the training current objective; planning of actions directed to the objective achievement [17].

By setting a sequential unit control action at system input there can be obtained permissible exposure coefficients. The table column specifies control actions, the table line indicates control parameters and contains influence coefficient of such parameter exposure. Usually the operator chooses control actions depending on their influence on parameter which is required to be changed [18]. For this purpose the greatest module coefficient is revealed in a line specifying this parameter values. Exposure value is determined by using the formula (16): 


$$
w_{j \mathrm{req}}=\frac{x_{t r e q}}{q_{t j}},(16)
$$

where $X_{\text {treq }}$ means a required change of the target parameter;

$q_{t j}$ is a coefficient of perturbations influence on a target.

Probable value of an exposure is determined with due consideration of a reserve for exposures. If there are parameters beyond the limits, then the exposure, which compensates an impermissible deviation, must be determined. The generalized algorithm of training with an application of situational model is given in [19].

\section{Results and Discussion}

The paper considers issues of heating power station process models development for the operators training systems. Based on results of the studies, a mathematical model was worked out describing control processes in heat power plants technological complex units considering interrelations between process variables in deviations in a real time mode. There was developed a system of decision-making at operator's training process control during heat power station units control both in nominal and emergency modes.

By comparison of mathematical model results, operating equipment test results [21-26] and performance data [2730] there was determined a static simulation error based on the main parameters: «steam temperature - feed water consumption». Static error does not exceed a limit defined by the formula (9).

Models accuracy was checked by comparison of model and boiler controlled parameters. In the course of the model accuracy check there were used the real processes running at heat power plants, as well as were considered cases of simplifying approximations applied at simulator models development. Aperiodic elements of the first and second order and of the first order with a time-lag were used for accuracy estimation.

Approximation of the table data with an application of formula (13) by a least-squares method produce values $\varepsilon$ $=0.8 ; \sigma=0.33$. Comparison of calculations and the experiment results confirm a hypothesis about applicability of dependence (13) at value $\alpha=0.05$.

"Input temperature - output temperature" transfer functions were applied at $T_{B}=1 ; \xi=10$ for determination of simulated characteristics. Values of $T_{M}$, which show change in the transition process time scale, were assumed to be 0.2 ; $0.6 ; 1 ; 1.4 ; 1.6$ respectively. A transient response $h=1-0.003 \mathrm{e}^{-6.02 T}-0.99 \mathrm{e}^{-0.08} \mathrm{~T}$ was applied as a standard.

Response curves were approximated as a series connection of a distance-velocity lag and an aperiodic link of a second order. Real processes were used as standards; there were also considered different cases of simplifying approximations used for simulator models development.

A combined impact of a time scale change and deviations in forms of monotonous processes were studied for the purpose to simplify a standard description by depression of differential equations.

Fig. 4 demonstrates examples of the processes used in the above described tests on one controlled parameter dynamics discrimination:
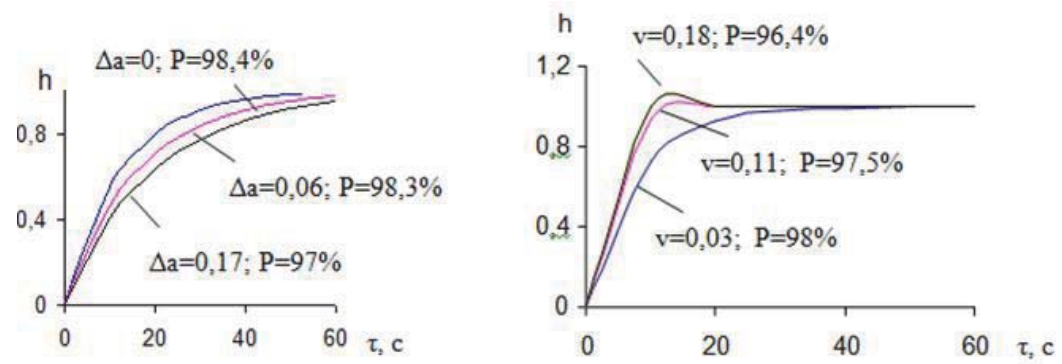

Fig. 4. Examples of processes used in tests on one controlled parameter dynamics discrimination: (a) change only in the standard process time scale; (b) change in the standard process time scale and form, $\mathrm{P}$ is a test adequacy ratio.

The developed software program complex can be used:

- $\quad$ as a part of a full-scale simulator for training of the cross-connection heat power plant personnel;

- principal provisions and mathematical models can be used at implementation of the project for heat power plant reconstruction;

- for development of setting complexes of real microprocessor control structures allowing to adjust control algorithms in conditions of a presetting training area. 


\section{Conclusion}

There was developed a mathematical model which describes control processes in heating power station units and considers interrelations between performance variables in deviations in real time. An algorithm of a steam generator process control model was worked out. The mathematical model and the object were tested for adequacy by statistical methods based on test data.

\section{Acknowledgment}

The author expresses its deepest appreciation to the lecturers of Almaty University of Power Engineering and Telecommunication for their support rendered in the course of this work implementation.

\section{References}

E.K. Arakelyan, V.P. Zverkov, V.F. Kuzishin, S.I. Magid. Peculiarities of computer-based simulators construction for complex trainings of heat power plant watch operating personnel, Teploenergetika 1 (2000) 42-49.

A.P. Ivanov, N.D. Mikheikina, T.B. Sizova. Software for dynamic simulation of thermohydraulic processes in steam boilers, Teploenergetika 10 (1998) 13-19.

A.S. Rubashkin. Computer-based training simulators for operators at thermal power stations, Teploenergetika 10 (1995) 38-46.

O.K. Shashkov. About principles of heat network computer-based simulators development, Teploenergetika 7 (1999) 37-39.

M.V. Sidulov, V.A. Martynov, N.Yu. Kudryavtsev. Mathematical simulation and optimization of HPP operation modes, Teploenergetika 10 (1993) $21-25$.

A.P. Ivanov. Software for the process dynamic simulation of heat power units at deep perturbing actions, Teploenergetika 10 (1988) 36-38.

S.I. Magid, I.P. Gerjoi and etc. A simulator for training of $250 \mathrm{mWt}$ heating power-generating unit operators, Electricheskie stantsii 8 (1984) $22-25$.

A.Ya. Frenkel, L.L. Maximova. Non-linear model of HPP steam generator zone with distributed constants and radiation heat supply, Teploenergetika 9 (1975) 41-46.

A.S. Rubashkin, V.L. Verbitskyi, V.A. Rubashkin. Methods of power equipment operation simulation, Teploenergetika 8 (2003) 44-48.

S.G. Maksimovitch. Formalized task for training exercise scenario in automated training system, in : Simulation in training systems: collection of scientific papers -Kiev: Naukova dumka (1990) 79-85.

Ye.P. Serov, B.P. Korolkov. Dynamics of steam generators. 2nd ed. Moscow: Energoizdat; 1981.

V.I. Plutinskyi, V.V.Okhotin. Methods for evaluation of accuracy of dynamic models for power generating unit simulators, Teploenergetika 10 (1985) 19-22.

Baimatayeva Sholpan M. ,Yu. V. Shevyakov. Study of Models for Heating Power Station Operator Training Systems. Journal of Energy and Power Engineering. Volume 7, Number 1, January (2013) 162-167.

Kuznetsov N.V., Mitor V.V., Dubovskyi I.Ye., Karasina E.S., Rubin M.M., Blokh A.G. and etc. Heat calculations for steam generating units. Moscow: Energy publishing house; 1973.

Yu. V. Shevyakov, Sh. M. Baimatayeva. Software and hardware complex for heat power station operator training systems, in: Proceedings of Scientific-Practical Conference, KazNTU, Almaty, 2008, pp. 409-413.

Sh. M. Baimatayeva. Situational simulator for operator training, Poisk, Almaty (3) (2005) 278-281.

Sh. M. Baimatayeva. Unit management procedures for system of operator training using expert system representation, in: Proceedings of the 10th International Conference "Energy-ecology-economy 2011", High Tatras, 2011, pp. 71-72.

A.F. Dyakov, S.D.Garbar. Simulation of operator's action plan development, Electricheskie stantsii 10 (1986) 9-11.

Sh. M. Baimatayeva, Yu. V. Shevyakov. Decision-making system for educational and training purposes, Poisk, Almaty (1) (2008) $274-278$.

Sh. M. Baimatayeva, Yu. V. Shevyakov. Simulation of thermal processes in a simulator steam generator for personnel training, Scientific news of Kazakhstan, Almaty (1) (2005) 99-103.

I.D. Lisanskyi, L.A. Pugachev, S.M. Svirskyi. Dynamic characteristics of steam temperature control sections in TPP-200 type boiler, section 800 mW. Teploenergetika 6 (1970) 8-11.

P. L. Suller, A.F. Romanov. Experimental dynamic characteristics of a direct-flow supercritical boiler, Teploenergetika 5 (1972) 56-61.

R. D. Tsiptsyura, V.E. Dulembo, B.P. Dyade. A simulator for training of power generation unit operators. Power engineering and electrification 3 (1975) 39-41.

A.T. Lebedev, A.V. Kondrashin, Yu.S. Tverskoi, V.N. Gushlo. Statistical characteristics of burning perturbations at different boiler operating modes, Teploenergetika 5 (1972) 77-79.

I.G. Polumordvinova, A.G. Chernov. Experimental and analytical study of dynamics in a steam generator with cyclone burners with due consideration of heat transfer variation depending on burner altitude, Teploenergetika 1 (1975) 30-34.

I.I. Aizenshtat, I.G. Polumordvinova, Ye.P. Feldman. Method of calculation of boiler superheat sections dynamic characteristics. Proceedings of Central Boiler and Turbine Institution (CKTI), Vol. 15, 1976.

A.L. Romanchik and others. Development of simulator operational characteristics. Nuclear steam power plant -2 engineering report, Almaty, 1990.

S.M. Magid. Section simulator of BKZ-420 type boiler water-steam circuit. Almaty, 1990.

S.M. Magid and others. Development of logical-dynamic model of BKZ-420-140-7c type boiler and PT-80-130 type turbine for a simulator training center Alma-AtaEnergo. Engineering report, Almaty, 1990.

S.M. Magid and others. Development of basic materials and work study at establishment of the Heating power station-2 staff training center. Engineering report, Almaty, 1989 